Saudi Journal of Biomedical Research

Abbreviated Key Title: Saudi J Biomed Res ISSN 2518-3214 (Print) |ISSN 2518-3222 (Online) Scholars Middle East Publishers, Dubai, United Arab Emirates Journal homepage: http://scholarsmepub.com/sjbr/

Review Article

\title{
Anemia and Women Subfertility
}

Mohammed Omer Mohammed Hussein*, Mohammed Abdelgafoor Abdelgadir, Mosab Nouraldein Mohammed

Banoon Fertility Center, Sudan

DOI: $10.36348 /$ sjbr.2019.v04i12.003

| Received: 05.12.2019 | Accepted: 18.12.2019| Published: 22.12.2019

*Corresponding author: Mohammed Omer Mohammed Hussein

\section{Abstract}

Anemia is a condition in which the blood hemoglobin (the main oxygen-transporting protein in red blood cells) level is under the minimum extreme of the reference range for the age and gender of the person .It is the most common hematological disorder affects millions of women at reproduction period around the globe that may influence in different phases of their socioeconomic lifestyle. It occurs due to increase red blood cell destruction or due to bone marrow failure to produce adequate number of blood cells. Iron deficiency anemia is the most common type of anemia noticed among women during their reproductive age, while other types are less common. Pernicious anaemia and folate deficiency are recognized to cause secondary infertility. Any attempts to treat infertility or to even establish preliminary investigations should be delayed until anaemia is treated. Management of anaemia itself may resolve the infertility problems and should be taken as a first line treatment in all cases.

Keywords: Anemia, women, subfertility.

Copyright @ 2019: This is an open-access article distributed under the terms of the Creative Commons Attribution license which permits unrestricted use, distribution, and reproduction in any medium for non-commercial use (NonCommercial, or CC-BY-NC) provided the original author and source are credited.

\section{INTRODUCTION}

Anemia - a state in which hemoglobin $(\mathrm{Hb})$ level and/or red blood cell (RBC) count are lower than normal and inadequate to meet a person's physiological requirements [1]. Internationally, anaemia influences 1.62 billion person (95\% CI: $1.50-1.74$ billion), which stands for $24.8 \%$ of the population (95\% CI: $22.9-$
$26.7 \%$ ). The maximum prevalence is in preschool-age children $(47.4 \%, 95 \%$ CI: 45.7-49.1), and the lowly prevalence is in men $(12.7 \%$, 95\% CI: 8.6-16.9\%). However, the population cluster with the most number of persons influenced is non-pregnant women (468.4 million, 95\% CI: 446.2-490.6).

Table-1: Showed Global anaemia prevalence and number of individuals affected

\begin{tabular}{|l|l|l|l|l|}
\hline \multirow{2}{*}{ Population group } & \multicolumn{2}{|l|}{ Prevalence of anemia } & \multicolumn{2}{l|}{ Population affected } \\
\cline { 2 - 5 } & Percent & $\mathbf{9 5 \%}$ CI & $\begin{array}{l}\text { Number } \\
\text { (millions) }\end{array}$ & 95\% CI \\
\hline Preschool children & 47.4 & $45.7-49.1$ & 293 & $283-303$ \\
\hline School-age children & 25.4 & $19.9-30.9$ & 305 & $238-371$ \\
\hline Pregnant women & 41.8 & $39.9-43.8$ & 56 & $54-59$ \\
\hline Non-pregnant women & 30.2 & $28.7-31.6$ & 468 & $446-491$ \\
\hline Men & 12.7 & $8.6-16.9$ & 260 & $175-345$ \\
\hline Elderly & 23.9 & $18.3-29.4$ & 164 & $126-202$ \\
\hline Total population & $\mathbf{2 4 . 8}$ & $\mathbf{2 2 . 9 - 2 6 . 7}$ & $\mathbf{1 6 2 0}$ & $\mathbf{1 5 0 0 - 1 7 4 0}$ \\
\hline
\end{tabular}

Source de Benoist B et al., eds. worldwide prevalence of anaemia 1993-2005 WHO Global Database on Anaemia Geneva, World Health Organization, 2008 [2]

To attain sufficient tissue perfusion--the outcome of flow, cardiac output, and oxygen-carrying capacity hemoglobin level must be fairly stable. Tissues deprived of oxygen are hypoxic and incur an "oxygen debt." That debt, an overuse of oxygen, needs a phase of raised oxygen delivery to permit repletion.
Universal oxygen release is recognized as the outcome of cardiac output and arterial oxygen content. Cardiac output is synchronized by preload, contractility, and following load, while oxygen content is estimated chiefly by hemoglobin concentration and, to a lesser degree, by the quantity of oxygen saturation. For 
practical aims, the association between cardiac out- put and arterial oxygen content is assumed to be linear over the normal clinical ranges; it most likely is not linear at the physiologic limits of endurance [3].

\section{Categories of Anaemia}

There are numerous kinds and classifications of anaemia. The incidence of anaemia is owing to the different red cell imperfections such as production failing (aplastic anaemia), maturation imperfection (megaloblastic anaemia), imperfections in hemoglobin production (iron deficiency anaemia), hereditary defects of hemoglobin maturation (thalassaemia) or due to the synthesis of abnormal hemoglobin (haemoglobinopathies, sickle cell anaemia and thalassaemia) and destruction of red cells (hemolytic anaemias) [4].

\section{Aplastic anemia}

"Anemia" derives from early capability to measure red blood cells in a hematocrit. The majority patients have pancytopenia, with reduced platelets and white blood cells. "Aplastic" refers to the inability marrow to form blood, the end organ effect of diverse pathophysiologic mechanisms. Historically, recognition of aplastic anemia was post-mortem, and the biopsy stays basic to diagnosis. Up

Till now a apparently empty bone marrow may be completely capable of supporting normal hematopoiesis. Equally, bone marrow failure can happen with normally cellular marrow, like in the myelodysplastic syndromes (MDS) and paroxysmal nocturnal hemoglobinuria (PNH) [5].

Three basic Pathophysiologies cause the pathology of an "empty" marrow.

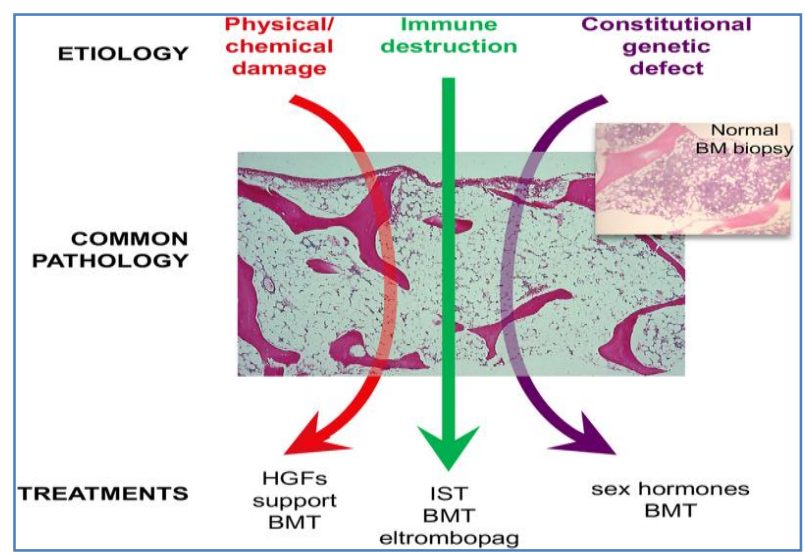

Fig-1: Pathophysiologies of aplastic anemia. The common pathology of the bone marrow replaced by fat can result from chemical or physical damage (iatrogenic; benzene); immune destruction (mainly $\mathbf{T}$ cells); and as a constitutional defect in genes important in maintenance of cell integrity and immune regulation. HGF=hematopoietic growth factors; $\mathrm{BMT}=$ bone marrow transplantation; IST=immunosuppression [5].

\section{Megaloblastic anemia}

Megaloblastic anemias are a kind of disorders recognized by the existence of distinguishing morphological features of the rising red cells in the bone marrow. The reason is frequently shortage of either cobalamin or folate, but megaloblastic anemia may occur due to inherited or acquired abnormalities affecting the metabolism of these vitamins or because of defects in DNA synthesis not related to cobalamin or folate. Macrocytosis is found in $2.5-4 \%$ of adults who have a routine complete blood count. In up to $60 \%$ of cases, macrocytosis is not associated with anemia; though, isolated macrocytosis should constantly be examined. Macrocytosis with no anemia may be a sign of early folate or cobalamine shortage, as macrocytosis headed progress of anemia. The average Indian vegan diet is lacking cobalamine [6].

\section{Iron deficiency anemia}

It is attributed to a prolonged negative imbalance between an individual's dietary intake of iron and their body's physiological need. Many non modifiable and modifiable factors exert an influence on an individual's iron balance, either in combination or alone, varying from Sociodemographic characteristics (including the person's age, gender, marital status, level of education, income, and ethnicity) to the amount and quality of the food and beverages they consume, their mental and physical health, the medication they take, any abnormalities they have, and their genetic makeup [7].

\section{Thalassemia}

It is the most frequent genetic blood disease in the world and varies in dissimilar population cluster in the world. World Health Organization (WHO) estimates that at least $6.5 \%$ of the world populations are carries of dissimilar inherited disorders of hemoglobin. The patients suffering from beta-Thalassemia major and $\mathrm{Hb}$ E/ beta-Thalassemia do not live for more than 5 years without blood transfusion. It (also known as Mediterranean anemia, Cooley's anemia, BetaThalassemia or Alpha-Thalassemia) is a genetic blood disorder exaggerated by an atypical form of hemoglobin blood disorder is the most frequent inherited single gene disorder in the world. This specific type of blood disease results in extreme breakdown of red blood cells which in turn leads to anemia [8].

\section{Sickle cell anemia}

It is the aftermath of a mutation in the gene for [3-globin, a subunit of adult hemoglobin A (HbA) (Figure 1). An adenine (A) to thymidine (T) substitution in codon 6 of the 1S-globin gene (GAG--->GTG (GUG in mRNA) specifies the synthesis of the sickle $\mathrm{t} 3$-globin chain (13 s or $\sim 6$ (A3) glutamic acid to lysine). HbS $((\mathrm{x} 2 \sim 2 \mathrm{~s})$ has the unique property of polymerizing when deoxygenated. Sufficient $\mathrm{HbS}$ polymer within the erythrocyte evokes the cellular injury responsible for the phenotype of sickle cell disease [9]. 


\section{Hemolytic anemia}

Hemolysis is the breakdown or removal of red blood cells from the circulation before their normal life span of 120 days. While hemolysis can be a lifelong asymptomatic condition, it most often exists as anemia when erythrocytosis cannot match the pace of red cell breakdown. It is also can manifest as jaundice, cholelithiasis, or isolated reticulocytosis.

There are two mechanisms of hemolysis. Intravascular hemolysis is the breakdown of red blood cells in the circulation with the release of cell contents into the plasma. Mechanical trauma from a destructed endothelium, complement fixation and activation on the cell surface, and infectious agents may induce direct membrane degradation and cell damage.

The more common extravascular hemolysis is the removal and damage of red blood cells with membrane alterations by the macrophages of the spleen and liver. Circulating blood is filtered continuously through thin-walled splenic cords into the splenic sinusoids (with fenestrated basement membranes), a spongelike labyrinth of macrophages with long dendritic processes. A normal 8-micron red blood cell can deform itself and pass through the 3-micron openings in the splenic cords. Red blood cells with structural alterations of the membrane surface (including antibodies) are unable to traverse this network and are phagocytosed and destroyed by macrophages [10].

\section{Pernicious anemia}

It is the most widespread reason of vitamin $\mathrm{B}_{12}$ deficiency. Vitamin $B_{12}$ insufficiency has several reasons; the term "pernicious anemia" applies only to the condition accompanied with chronic atrophic gastritis. A current population survey revealed that 1.9 percent of individuals more than 60 years old have undiagnosed pernicious anemia. Previous studies revealed that pernicious anemia is constricted to Northern Europeans. However, subsequent studies have documented the disease in black and Latin-American subjects, with an earlier age of onset in black women. Although the disease is silent until the end stage, the underlying gastric lesion can be predicted many years before anemia develops. Successful treatment of the anemia with cooked liver suggested that it was caused by the lack of an extrinsic factor that was found in liver (later identified as vitamin $\mathrm{B}_{12}$ ) and an intrinsic factor in gastric juice. Although pernicious when first discovered, the disease is now controlled by treatment with vitamin $\mathrm{B}_{12}$. The discovery of a serum inhibitor of intrinsic factor (later found to be an autoantibody to intrinsic factor) and of autoantibodies to parietal cells laid the foundation for the immunologic explanation of the underlying gastritis that causes pernicious [11].
Fertility is the capability to establish a clinical pregnancy; the term infertility is used by some clinicians interchangeably with subfertility.

Globally, more than186 million people suff er from infertility, the bulk being inhabitants of developing countries. As the most influential negative prognostic factor of fertility is rising women's age at conception [12].

Infertility is a clinically widespread disease, happening in approximately $10 \%$ of women of childbearing age. Studies have confirmed that there is an intimate association between deficiency in trace elements and mysterious infertility in females. A recent epidemiological observational study suggested that women without iron supplementation had a higher risk of ovulatory infertility compared with women who used an iron supplement [13].

The belief, based on general experience, has been formerly expressed that patients in the childbearing age group with Addisonian pernicious anaemia may be infertile. While it is not widespread in the childbearing age group, our findings do not concur with the lately expressed view that this type of anaemia 'is very rare under the age of 40'. We would powerfully recommend that (a) serum B12 and folate assays are incorporated as routine measures in the examination of all infertile female patients [14].

Women with SCD have extra risk factors that can impact their aptitude to conceive and comprise chronic inflammation, oxidative stress, transfusionrelated hemochromatosis, and ovarian sickling leading to ischemia and reperfusion injury to the ovary. This is an fast growing area of research, as little is known about the estimated prevalence of infertility and fecundity in women with SCD. Additionally, using contraception while on hydroxyurea therapy during reproductive years and discontinuing hydroxyurea therapy for family planning and during pregnancy are strongly recommended. Previous studies have elucidated that women with SCD have additional risk factors for infertility, including the chronic use of disease-modifying therapies (regular blood transfusion therapy and hydroxyurea), NSAIDs, and opioids. The only curative options for SCD are HSCT and gene therapy, which often involves conditioning regimens containing alkylating agents and total body irradiation that contribute to infertility and primary ovarian insufficiency. Therefore, it is recommended that clinicians refer patients to a reproductive endocrinologist to discuss fertility preservation options including surrogacy, even for those who are ambiguous about having future children prior to HSCT or gene therapy [15].

Fanconi anemia (FA) is an uncommon autosomal recessive disorder that belongs to the group 
of chromosomal unsteadiness syndromes. FA cells of all organ tissues are hypersensitive to DNA crosslinking agents and to oxygen, and have cell cycle anomalies. In the majority of mammalian species, the creation of ovarian oocytes is thought to cease after birth. Though, this principle has been faced by research representing that female gonad have regenerative action in juvenile and adult mice in vivo. Johnson et al. published a hypothesis postulating that fertility revival after transplant might be the result of germ line stem cells supplied by the donor bone marrow.

Analysis of the genetic origin of the child of one of our FA patients who consented to a genetic analysis among mother, daughter and donor. These results showed clearly the genetic relationship between the transplanted patient and her daughter, excluding the possibility of germ cell transmission from the donor. This suggested that fertility recovery after BMT could only result from incomplete depletion of the ovarian follicle reserve [16].

\section{DISCUSSION}

Anemia is a very common disorder which affects huge numbers of women, particularly in developing countries due to many factors such as nutritional, microbial and even hereditary reasons, further more inadequate health care service; certainly in rural areas enhance the development of its complications. According to the estimation of world health organization, the most group influenced by anemia was non pregnant women (468 million), and these open doors to look at anemia as possible risk factor for women sub fertility. Furthermore hemochromatosis due to recurrent blood transfusion, susceptibility to infection and even behavior abnormality and depression. According to literature iron deficiency, megaloblastic, pernicious, aplastic, hemolytic anemia and Thalassemia may affect women fertility with different mechanisms.

\section{CONCLUSION}

Extensive studies must be done to investigate the relation between subfertility and anemia of different etiologies, including patients from different regions, ethnic and age groups.

\section{RECOMMENDATIONS}

Anemia management must be the first step to resolve subfertility issues of anemic women.

\section{REFERENCES}

1. Chaparro, C. M., \& Suchdev, P. S. (2019). Anemia epidemiology, pathophysiology, and etiology in low-and middle-income countries. Annals of the New York Academy of Sciences, 1450(1), 15.
2. De Benoist, B., Cogswell, M., Egli, I., \& McLean, E. (2008). Worldwide prevalence of anaemia 19932005; WHO Global Database of anaemia.

3. Schneider, A. J., \& Oski, F. A. (1981). Transfusion nomogram: an application of physiology to clinical decisions regarding the use of blood. Critical care medicine, 9(6), 469-473.

4. Mukharjee, K. L. (1997). Medical laboratory technology. A procedure manual for routine diagnostic tests. Vol I-III.

5. Young, N.S. (2018). Aplastic Anemia. N Engl J Med, 379(17):1643-1656.

6. Srikanth, S. (2016). Megaloblastic anemia-A clinical spectrum and a hematological profile: The day-to-day public health problem. Medical Journal of Dr. DY Patil University, 9(3), 307.

7. Alzaheb, R. A., \& Al-Amer, O. (2017). The prevalence of iron deficiency anemia and its associated risk factors among a sample of female university students in Tabuk, Saudi Arabia. Clinical Medicine Insights: Women's Health, 10, 1179562X17745088.

8. Palit. (2012). Prevalence and correlation of thalassemia between LFT with age and sex. Journal of Basic and Clinical Pharmacy, 3(4).

9. Steinberg, M. H. (1998). 6 Pathophysiology of sickle cell disease. Baillière's Clinical Haematology, 11(1), 163-184.

10. Gurpreet, Dhaliwal, M.D., Patricia, A., Cornett, M.D., \& lawrence M. Tierney, JR., M.D. hemolytic anemia.(2004). American family physician, JUNE 1, 69(11), 2599-2606.

11. Toh, B. H., van Driel, I. R., \& Gleeson, P. A. (1997). Pernicious anemia. New England Journal of Medicine, 337(20), 1441-1448.

12. Mélodie, V.B., Clinical Biochemistry. (2018). https://doi.org/10.1016/j.clinbiochem.2018.03.012.

13. Li, Y. Q., Cao, X. X., Bai, B., Zhang, J. N., Wang, M. Q., \& Zhang, Y. H. (2014). Severe iron deficiency is associated with a reduced conception rate in female rats. Gynecologic and obstetric investigation, 77(1), 19-23.

14. Hall, M., \& Davidson, R. J. (1968). Prophylactic folic acid in women with pernicious anaemia pregnant after periods of infertility. Journal of clinical pathology, 21(5), 599-602.

15. Ghafuri, D. L., Stimpson, S. J., Day, M. E., James, A., DeBaun, M. R., \& Sharma, D. (2017). Fertility challenges for women with sickle cell disease. Expert review of hematology, 10(10), 891901.

16. Nabhan, S. K., Bitencourt, M. A., Duval, M., Abecasis, M., Dufour, C., Boudjedir, K., \& Sanders, J. (2010). Fertility recovery and pregnancy after allogeneic hematopoietic stem cell transplantation in Fanconi anemia patients. haematologica, 95(10), 1783-1787. 